\title{
Heart failure in the young: Insights into myocardial recovery with ventricular assist device support
}

\author{
Eva Maria Javier Delmo ${ }^{1}$, Mariano Francisco del Maria Javier ${ }^{2}$, Dietmar Böthig ${ }^{3}$, Andre Rüffer $^{4}$, \\ Robert Cesnjevar ${ }^{5}$, Michael Dandel ${ }^{6}$, Roland Hetzer ${ }^{2}$ \\ ${ }^{1}$ Charité-Universitätsmedizin Berlin, Charité Research Organization, Berlin, Germany; ${ }^{2}$ Department of Cardiothoracic and Vascular Surgery, Cardio \\ Centrum Berlin, Berlin, Germany; ${ }^{3}$ Department of Cardiothoracic, Transplantation and Vascular Surgery, Hannover Medical School, Hannover, \\ Germany; ${ }^{4}$ Department of Pediatric Cardiology/Pediatric Cardiac Surgery, Universitäres Herzzentrum Hamburg GmbH, Hamburg, Germany; \\ ${ }^{5}$ Department of Cardiothoracic and Vascular Surgery, University Medical Center Erlangen, Erlangen, Germany; ${ }^{6}$ Department of Cardiology, Cardio \\ Centrum Berlin, Berlin, Germany \\ Contributions: (I) Conception and design: All authors; (II) Administrative support: None; (III) Provision of study materials or patients: EM Javier \\ Delmo, R Hetzer, R Cesnjevar, A Rüffer; (IV) Collection and assembly of data: All authors; (V) Data analysis and interpretation: D Böthig, M \\ Dandel; (VI) Manuscript writing: All authors; (VII) Final approval of manuscript: All authors. \\ Correspondence to: Eva Maria Javier Delmo, MD, MSc, PhD. Charité-Universitätsmedizin Berlin, Charité Research Organization, Virchowweg 10, \\ 10117 Berlin, Germany. Email: eva.javierdelmo@gmail.com.
}

Background: Data on ventricular unloading-promoted myocardial recovery and post-weaning outcome in children is scarce. We analyzed the weaning outcome in children with heart failure (HF) supported with ventricular assist device (VAD).

Methods: A multi-institutional data on VAD implanted in 193 children and adolescents with HF between April 1990 and November 2015 was reviewed. Among them, 25 children (mean age 3.4 3.0, range, 0.058-16.3 years, 15 females) were weaned from VAD. Etiology of HF were myocarditis ( $n=11)$, dilated cardiomyopathy (DCMP) $(n=7)$, ischemic HF ( $n=3)$, arrhythmogenic CMP $(n=1)$, post-correction of congenital heart disease $(\mathrm{CHD})(\mathrm{n}=1)$ and acute graft failure $(\mathrm{n}=1)$. Mean duration of HF before VAD implantation was $59.4 \pm 3$ days.

Results: Age, duration of HF, DCMP, cardiac arrest and duration of VAD are essential clinical characteristics to delineate who may have the potential to myocardial recovery. Echocardiographic parameters pre-implantation, during the final off-pump trial and during the post-explantation follow-ups revealed that LVEF, LVEDD and relative wall thickness (RWT) showed significant differences $(\mathrm{P}<0.001)$ among patients stratified by outcome to assess recovery. Presently, 21 (84.0\%) of the weaned patients are alive with their native hearts 1.3-19.1 years after VAD explantation. An additional weaned patient had HF recurrence 3 months post-weaning and was transplanted.

Conclusions: Post-weaning myocardial recovery and cardiac stability of children with HF from several etiologies supported with a VAD appears sustainable and durable. Young patients with short HF duration are more likely to recover. Absence of cardiac arrest, cardiac size, geometry and function may prospectively identify patients who may be likely to have myocardial recovery.

Keywords: Heart failure (HF); ventricular assist devices; myocardial recovery; heart transplantation

Submitted Feb 25, 2020. Accepted for publication Apr 21, 2020.

doi: $10.21037 / \mathrm{cdt}-20-278$

View this article at: http://dx.doi.org/10.21037/cdt-20-278 


\section{Introduction}

Although medical therapy has improved the survival and quality of life of children with heart failure (HF), those with cardiogenic shock and acute HF with underperfusion need mechanical circulatory support systems to maintain end-organ perfusion $(1,2)$. By unloading the ventricle with a ventricular assist device, complete myocardial recovery may be achieved. Until now, no factors are identified which predict recovery. Likewise, if recovery occurs, there is no guarantee that it lasts after ventricular assist device (VAD) explantation. Weaning of adults off VAD support has evolved and has been demonstrated that there can be long-term success (3-5). However, ventricular unloadingpromoted myocardial recovery and post-weaning outcome data in children is scarce. The potential for myocardial recovery after VAD support in children was asserted by our group in 1998 (6), in 1999 (7) and in 2018 (8). To provide updates, we analyzed the weaning outcome in children with HF supported with a VAD with regards to the recurrence of $\mathrm{HF}$ and the sustainability of myocardial recovery after VAD explantation. Moreover, we sought to determine the clinical characteristics and echocardiographic variables that may identify patients who may likely have myocardial recovery.

\section{Methods}

The Institutional Review Board approved this study and waived the need for patients' consent as the study itself was regarded as non-interventional and data protection standards were fulfilled. The authors had full access to and take full responsibility for the integrity of the data. All authors have read and agreed to the manuscript as written.

Review of medical records of children with HF in three German university pediatric cardiac centers identified 193 children supported with various VADs between April 1990 and November 2015. For homogeneity of the analysis, only patients $(\mathrm{n}=148)$ supported with a pneumaticallydriven, paracorporeal pulsatile flow pump (EXCOR Pediatric VAD, Berlin Heart GmbH, Berlin, Germany) were considered. For outcome comparison of VAD support, these children were stratified into three groups: children who had myocardial recovery; children who were bridged to orthotopic heart transplantation (OHT); and children who succumbed while on VAD support.

The weaning outcome and durability of myocardial recovery post-VAD explantation in 25 children with $\mathrm{HF}$ supported with VAD were the main subjects of this study.
Clinical characteristics (age, gender, duration of HF before VAD implantation, presence of cardiac arrest, implantation and duration of ECMO support prior to VAD, duration of VAD support) and echocardiographic data (cardiac size, geometry and function) were reviewed. This cohort was followed up until April 2017.

\section{Timing of implantation, choice of pump sizes and techniques}

The indications for VAD support, choice of pump size and implantation techniques have been previously reported by our group $(1,9,10)$.

The primary goal was to implant a left VAD (LVAD). However, when patients failed to stabilize with a LVAD after coming off cardiopulmonary bypass and who had signs of refractory right ventricular (RV) failure, a right VAD (RVAD) was added (9).

Biventricular VAD (BVAD) implantation in infants and small children has been challenging because of the small pericardial space, and positioning the four cannulae after reconstructive operations could become complex. As we gained more experience in VAD implantation, it became apparent that cannulation of the left ventricular $(\mathrm{LV})$ apex does not only improve $\mathrm{LV}$ emptying but also supports the $\mathrm{RV}$ as well; thus, biventricular support became necessary only in patients with more advanced HF.

We have fully described the post-VAD anticoagulation management in our previous reports $(1,9,10)$.

Pump exchange was done for any thrombus formation in the left pump or in the left-side cannulae and thrombus of more than a few millimetres in size or free-floating thrombi of any size in the right pump and cannulae (9).

A prophylactic antibiotic with anti-staphylococcal coverage is administered in the perioperative phases.

Children are mobilized as soon as their condition allows. Infants and small children dependent on the powerful, stationary IKUS driver are kept in hospital, whereas older children ( $\geq 15$ years old, $n=11$ ) who could operate the portable EXCOR Pediatric VAD driver are discharged home whenever possible, and go to school with the device.

Immediately after VAD implantation, HF therapy (-blocker, an angiotensin converting enzyme inhibitor and spironolactone) was started in all children. Other drugs (diuretics, ivabradine, digoxin) are used as necessary. 


\section{Echocardiographic assessment of cardiac dimensions, geometry and function}

Daily transthoracic echocardiography (TTE) is performed routinely in children kept in the hospital, and weekly in children who were discharged with the VAD, to monitor cardiac function and screen potential weaning candidates. Echocardiogram reports were reviewed to determine the change in cardiac function, dimensions and geometry while on VAD support. Cardiac parameters considered to assess cardiac recovery included: (I) LVEDD measured in the antero-posterior plane as the maximal diastolic diameter between the septal to posterior wall endocardium at the level of the papillary muscle tips; (II) posterior wall thickness (PWT) measured in diastole between the papillary muscles at the level of their tips: (III) end-diastolic LV relative wall thickness (RWT) measured in the parasternal long-axis view at the base of the $\mathrm{LV}$ using the formula RWT $=2 \times$ PWT/LVEDD; (IV) LV fractional shortening (FS) $\geq 15 \%$ measured in parasternal view at the base of the LV; and (V) LVEF. Values obtained, expressed as z-scores to adjust for the effect of body size and age, are compared among the three groups to assess which variables could predict myocardial recovery during VAD support.

\section{Indications for weaning and VAD explantation}

\section{Criteria for weaning}

TTE is the primary imaging modality for selection of weaning candidates and is mandatory for weaning decisions. Miera et al. (8) has recently published the Berlin weaning protocol for treatment during VAD support and evaluation of cardiac function during pump stops, which was developed and implemented in 2005.

Generally, the first step is selection of potential weaning candidates: those with sinus rhythm, normal LVEDD, improvement of $\mathrm{LV}$ wall motion, none or $\leq$ grade 1 mitral and/or aortic valve regurgitation, no RV dilation and no or $\leq$ grade 2 tricuspid regurgitation (TR) during full LVAD support. Progressive increase in duration and frequency of aortic valve openings during unchanged pump rate also indicated improvement of $\mathrm{LV}$ contractile function. Provided that there is no bleeding nor a recent thromboembolic event and anticoagulation was within target parameters, 30 $50 \mathrm{IU} / \mathrm{kg}$ of unfractionated heparin is administered prior to VAD stops. In such potential weaning candidates, to verify whether complete unloading interruption trials were possible and reasonable, it is useful to perform TTE-guided stepwise pump rate reductions to $30 / \mathrm{min}$ (short 5 -minute pump stops) and then completely stopped for approximately 5 minutes, before evaluation of cardiac function during complete interruptions of the LVAD support. If stepwise pump rate reductions provoke symptoms (dizziness, sweating, etc.) and/or significant cardiac arrhythmia, LVEDD increases beyond the normal range, and/or the RV showed morphological and functional instability (increasing TR, RV dilation with reduced output), the patient was deemed not a weaning candidate and unloading interruption trials were not indicated.

Echocardiographic assessment of recovery was mostly based on data obtained at rest during repeated shortterm interruptions of LVAD support (off-pump trials). Hemodynamic and echocardiographic parameters were obtained at baseline, after reduction of the pump rate to $30 / \mathrm{min}$, and during pump stops after 10, 20, and 30 minutes. During an off-pump or pump turn-down trial, this stepwise echocardiographic assessment in the course of repeated shortterm $(\sim 5 \mathrm{~min})$ unloading interruptions were deemed safer than one or two longer-lasting (15-20 min) interruptions. Nonetheless, a 15-20-minute complete interruption of the LVAD support in the operating theatre (without inotropic or ventricular preload and afterload support) was mandatory for the final echocardiographic evaluation concerning the decision in favour or against LVAD explantation.

Assessment of myocardial recovery in children with BVAD implantation was similar to that after LVAD implantation, but often more challenging. This necessitates stops of both pumps. The RV pump is stopped $\sim 30 \mathrm{~s}$ earlier than the LV pump. The first echocardiographic and hemodynamic evaluation was conducted 5 minutes after VAD stops while on full LVAD support. During the off-pump trials, special attention is paid to the RV size, geometry and load, and presence of TR. Increases in RV end-diastolic diameter short-/long-axis ratio, TR and right atrial pressure (RA) $\geq 10 \mathrm{mmHg}$, indicates worsening of RV function.

In long-term LVAD recipients with additional RVAD support, selection of patients for RVAD removal is usually started 48-72 hours after RVAD implantation. Weaning candidates are clinically stable patients with adequate renal, hepatic, pulmonary and neurologic functions, with regression of $\mathrm{RV}$ dilation, improvement of $\mathrm{RV}$ wall motion and no significant TR. Weaning is started with gradual reduction of $\mathrm{RV}$ pump-flow to $2 \mathrm{~L} / \mathrm{min}$ at $0.5-\mathrm{L} /$ day increments under transthoracic echocardiographic guidance and monitoring of hemodynamic responses [RV pump- 
flow reduction below $2.0 \mathrm{~L} / \mathrm{min}$ (cardiac output/BSA) for a prolonged period is not recommended]. Additional reduction of the PVR by a phosphodiesterase-5 inhibitor has been beneficial for RV recovery assessment. Septumshifting toward the LV (sign for RV dilation), increase in TR and RA pressure and decrease in cardiac index or LVAD-index during RVAD flow reduction are major signs for insufficient RV recovery, and necessitates interruption of the weaning process and return to initial right VAD settings. If echocardiographic and hemodynamic measurements remained stable during moderate reduction of RVAD flow, a short (5-10 $\mathrm{min}$ ) interruption of RV support was necessary for the actual assessment of RV recovery. If echocardiographic-measured parameters remained stable and hemodynamic measurements continued to remain within the normal range, a stable RV recovery was very likely.

Weaning candidate selection and assessment of RV recovery in isolated right VAD recipients is similar to the assessment of RV recovery in long-term biventricular VAD recipients or LVAD recipients with an additional RVAD. TTE focuses especially on possible changes in atrial and ventricular septum position and RV end-diastolic dimensions and short-/long-axis ratio, as well as on changes in tricuspid annular plane systolic excursion (TAPSE).

Special attention has been paid to TR and to the continuous-flow Doppler-derived pressure gradients between the RV and RA. Serial velocity-time integral measurements obtained from PW-Doppler signals in the LV outflow tract were useful for non-invasive monitoring of stroke volume and cardiac index stability. If hemodynamic measurements remained within the normal range and echocardiographic parameters remained stable during moderate reduction of the right VAD flow, a short (5-10 min) interruption of RV unloading is performed. If echocardiographic-measured parameters remained stable during that complete interruption of RV unloading, RV recovery appears sufficient to allow right VAD explantation. With stable hemodynamics, LVAD pump rate is reduced and subsequently stopped. To prevent clot formation, the VAD is set to pump twice/minute during the procedure; thereafter, baseline VAD settings were reset at the end of the examination. No further attempt was made to reduce the mechanical support stepwise before explantation. The VAD was explanted within the subsequent days.

\section{VAD explantation}

VAD explantation was performed in the operating theatre. The pump flow was gradually decreased and echocardiographically-measured LV filling pressures were monitored. Weaning was performed with the pump being stopped for 20 minutes when the following criteria were achieved, both in patients with left VAD or BVAD support: (I) LVEDD <98th percentile (z-score <+2); (II) LVEF $\geq 45 \%$ (i.e., no more than mild LV dysfunction); (III) normotensive on milrinone only (no other inotropes); (IV) lactate $<3 \mathrm{mmol} / \mathrm{L}$; (V) LV end -diastolic pressure < $12 \mathrm{mmHg}$; (VI) resting cardiac index of $>2.8 \mathrm{~L} / \mathrm{min} / \mathrm{m}^{2}$; (VII) RWT of 0.27 ( $\mathrm{Z}$ score $<+2$ ), and (VIII) no RV dilatation, absence or mild aortic, mitral and tricuspid valve insufficiency.

After selection of weaning candidates, VAD explantation is performed as follows: (I) stepwise pump rate reductions under TTE monitoring, to verify whether complete unloading interruption trials were possible and reasonable before assessing the heart during complete interruptions of VAD support (possible if moderate reduction was supported by the patient without any symptoms; reasonable only if moderate reduction did not induce cardiac and hemodynamic changes which indicated that VAD explantation is too risky or even impossible); (II) off-pump trial with echo-examination at rest during several (2-4 times) short-time $(\approx 5-10 \mathrm{~min})$ interruptions of mechanical circulatory support (under additional anticoagulation therapy); (III) LV filling pressures-derived hemodynamic measurements during short-term VAD support interruptions; and (IV) final $\geq 15$ min long off-pump trial in the operating room with repeated simultaneous echocardiographic and hemodynamic measurements.

Stable central venous saturation and low pulmonary artery pressure during pump stops were likewise considered to be adequate prognostic parameters, as was normalization of BNP or NT-proBNP levels during the whole weaning phase. When hemodynamics and all aforementioned parameters remain stable during "off-pump" trials, VAD explantation is carried out.

\section{Follow-up}

Follow-up data were provided by written correspondence from the referring physicians. All patients operated from 1990 to 2015 had complete follow-up with series of echocardiograms. No patients were lost to follow-up.

\section{Statistical analysis}

Data was analyzed using IBM SPSS version 25.0 software (IBM Armonk, New York). Continuous variables were 
reported as means \pm standard deviation, median and range. Normal distribution of numeric variables was assessed with Kolmogorov-Smirnov test. Significant distribution differences within the three groups were further evaluated with Kruskal-Wallis test, Pearson $\mathrm{Chi}^{2}$-test or univariate analysis of variance (ANOVA), as appropriate. Influence of clinical characteristics and echocardiographic parameters on primary outcome were assessed by Chisquare analysis. A competing risk model considering the times on VAD, recovery, OHT and death was performed using the R-packages "mstate" by Putter, Fiocco, Geskus [2007] (R Foundation for Statistical Computing, Vienna, Austria. http://www.R-project.org/.) Within the multistate model, the function "probtrans" to examine age at VAD implantation, LVAD vs. BVAD, and the diagnosis group [dilated cardiomyopathy, myocarditis, postoperative HF or congenital heart disease (CHD)] by uni- and multivariate analysis as potential factors for factors for the transition from the state "on VAD" to the state "weaning" was applied. Event-free percentages were estimated and shown according to Kaplan-Meier estimates at $95 \%$ confidence interval. A $\mathrm{P}<0.05$ was considered statistically significant.

\section{Results}

Table 1 shows the demographic profile of 148 children (mean age $6.1 \pm 3.4$, median 4.4, range, $0.01-17.8$, years) with advanced HF supported with a VAD. There were 41 (27.7\%) infants ( $<1$ year, old) and $83(56.0 \%)$ were females. Etiologies of HF were DCMP ( $\mathrm{n}=76,51.3 \%)$, myocarditis $(\mathrm{n}=33,22.2 \%)$ and post-correction of CHD $(n=29,19.6 \%)$. Duration of HF before VAD support ranged from 1 day to 14 years. Forty-nine $(33.1 \%)$ had suffered cardiac arrest, $16(10.8 \%)$ of whom had initially ECMO support prior to VAD implantation. Left VAD was mostly $(\mathrm{n}=92,62.1 \%)$ implanted followed by BVAD ( $\mathrm{n}=55,37.1 \%)$. There was only one instance when a right VAD was implanted on a post-transplant patient with acute graft failure.

Table 2 shows the comparative detailed outcome of VAD support in 148 children. There were 25 (16.9\%) children who were successfully weaned off VAD support with complete myocardial recovery and discharged home. Seventy-eight $(52.7 \%)$ showed unattainable weaning on pump-stop trials; hence, eventually underwent OHT. Fortyfive $(30.4 \%)$ children succumbed while on support.

Table 3 describes the 25 children who had myocardial recovery. Mean duration of VAD support was $77.8 \pm 18.3$ (median 42, range 7-700) days. Figure 1 illustrates the time on VAD support before myocardial recovery was achieved. All patients who recovered were weaned within 155 days after VAD implantation, except 1 who recovered after 700 days. Four patients needed $\geq 100$ days of support before VAD explantation. In children with myocarditis $(n=11)$, median HF duration was 12 days (range, 3-30 days). Two of these children underwent cardiopulmonary resuscitation (CPR) and one had ECMO support before switching to VAD. VAD was explanted in 7 children with DCMP, whose HF duration ranged from 7-480 days, one of whom was resuscitated and initially supported with ECMO for 19 days. All of these patients had their ventricular cores biopsied to rule out myocarditis. The 16-year-old adolescent with acute graft failure was supported with RVAD for 78 days. She is still alive 11.7 years post-weaning. Among 3 children with ischemic HF weaned off VAD support 7, 12 and 13 days, respectively, one had HF recurrence 3 months post-weaning and underwent OHT. Unfortunately, she died 8.6 years later.

Mean follow-up duration is $9.0 \pm 7.1$ (median 7.8, range, 1.4-19.1) years. Freedom from HF recurrence is $96.0 \% \pm 3.9 \%$ (Figure 2). None of the weaned patients underwent repeat VAD implantation. Three (12.5\%) patients died 2.4, 3.3 and 15.6 years, respectively, postweaning. Cumulative survival is $84.0 \%$ (Figure 3).

To understand the underlying function- and geometryrelated variables in the myocardium which predisposes to recovery, changes in LVEF, FS, LVEDD and RWT preVAD implantation, during VAD support and after VAD explantation (the isolated RVAD support for a postoperative graft failure was excluded) were compared among the three groups (Table 4). Figure 4 shows the percent changes in $\mathrm{LV}$ end diastolic diameters between 3 time points: shortly before VAD implantation, shortly after VAD implantation and after VAD explantation for patients who had recovery and after heart transplantation for heart transplant recipients. Differences in LVEF, FS, LVEDD and RWT among the groups over these specified time points are highly significant $(\mathrm{P}<0.001)$. On univariate analysis, z-scores for LVEDD and LVFS were associated with normalization in patients who were weaned off VAD support.

Figure 5 is the stacked transition probabilities plot derived from the competing risks multistate estimation for time-related events of children with HF supported with VAD. The continuous lines show the estimations, the dashed lines their confidence limits. The vertical distance between a line and the line underneath shows the fraction of patients that can be expected to be in the state described in the figure at the time given on the $\mathrm{x}$-axis. To find the 
Table 1 Baseline characteristics of children with advanced heart failure supported with VAD

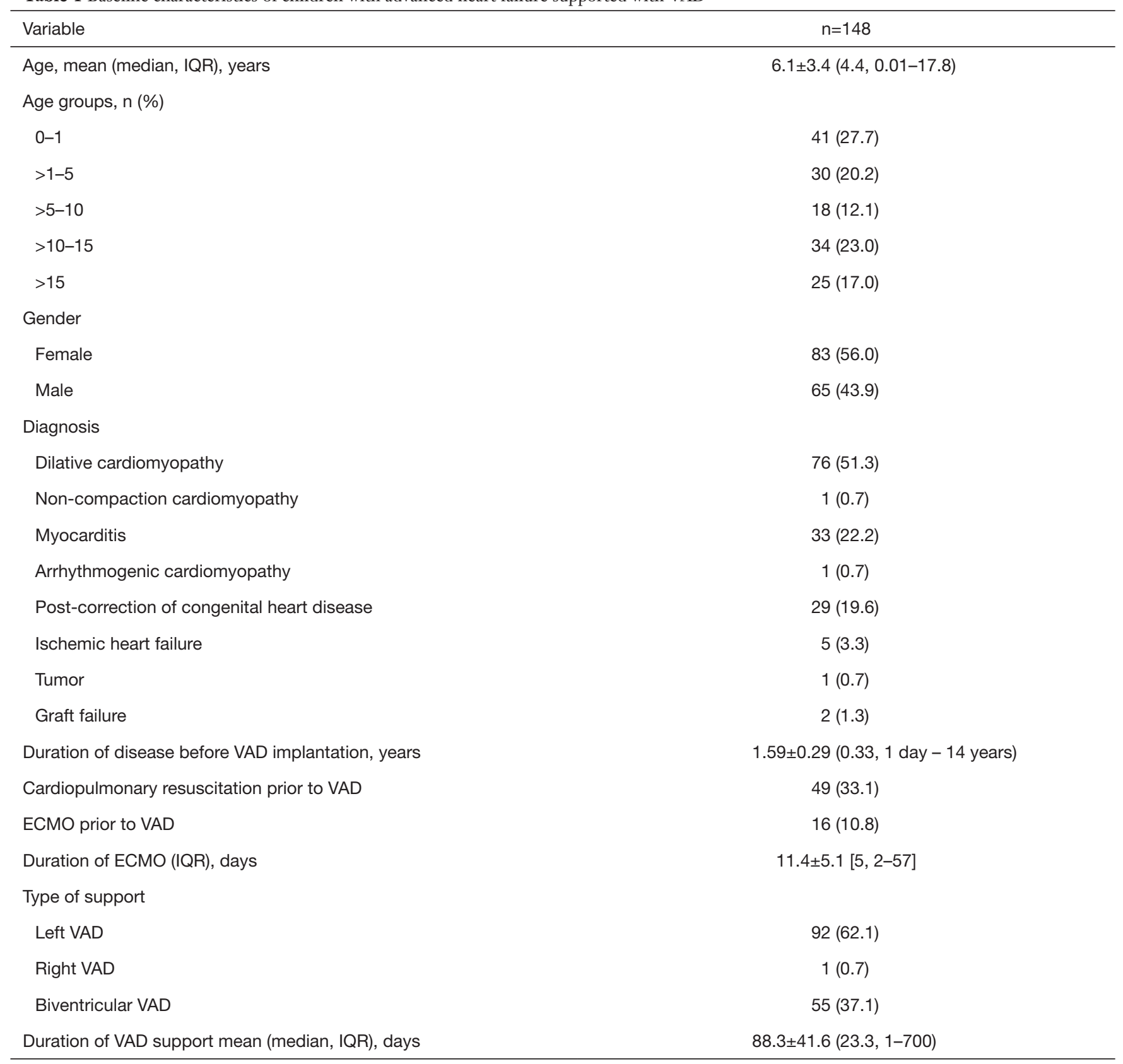

percentage of patients that corresponds to the vertical distance, shift the distance arrow to the bottom of the y-axison the bar top, the scale indicates the percentage of patients being in this state. For example, at 15 years about $13 \%$ of the children who needed VAD support were in the state of recovery. In effect, the competing risk analysis shows that the patients who could be weaned had a low risk for HF recurrence or death in the ensuing years. Presently, 21 (84\%) among the weaned patients are alive with their native hearts. All patients with DCMP remained free from post-weaning HF recurrence and all are alive (mean post-weaning survival time $7.8 \pm 4.6$, range $2.5-16.2$ years). That only one child had HF recurrence makes it impossible to identify children who might have been weaned inappropriately.

Multistate analysis showed that younger patients and patients with CHD had significantly better chances for 
Table 2 Univariate analysis of profile of children with advanced heart failure supported with VAD stratified according to outcome

\begin{tabular}{|c|c|c|c|c|}
\hline Variable & $\begin{array}{l}\text { Children who had } \\
\text { myocardial recovery }(n=25)\end{array}$ & $\begin{array}{l}\text { Children supported with } \\
\text { VAD as a bridge to heart } \\
\text { transplant }(n=78)\end{array}$ & $\begin{array}{l}\text { Children who died } \\
\text { on VAD }(n=45)\end{array}$ & $P$ value \\
\hline Age groups, $n(\%)$, years & & & & 0.066 \\
\hline $0-1$ & 12 & 14 & 15 & 0.026 \\
\hline$>5-10$ & 1 & 11 & 6 & 0.307 \\
\hline$>10-15$ & 3 & 20 & 11 & 0.308 \\
\hline$>15$ & 1 & 18 & 6 & 0.148 \\
\hline Diagnosis & & & & $<0.001$ \\
\hline DCMP & 7 & 56 & 13 & $<0.000$ \\
\hline Non-compaction CMP & 1 & 0 & 0 & 0.084 \\
\hline Myocarditis & 11 & 14 & 8 & 0.074 \\
\hline Arrhythmogenic cardiomyopathy & 1 & 0 & 0 & 0.084 \\
\hline Post correction of CHD & 1 & 5 & 23 & 0.084 \\
\hline Ischemic heart failure & 3 & 2 & - & $<0.001$ \\
\hline $\begin{array}{l}\text { Mean duration of ECMO support } \\
\text { (median, range), days }\end{array}$ & Range 6-19 & $10.2 \pm 5.7[5,2-31]$ & $12.0 \pm 3.8[5,2-57]$ & 0.568 \\
\hline Type of support & & & & 0.324 \\
\hline LVAD & 19 & 51 & 21 & 0.711 \\
\hline RVAD & 1 & 0 & 0 & 0.267 \\
\hline BVAD & 6 & 27 & 22 & 0.566 \\
\hline $\begin{array}{l}\text { Mean duration of VAD support (median, } \\
\text { range interquartile range), days }\end{array}$ & $\begin{array}{c}77.8 \pm 18.3 \\
{[42,7-700,15-81]}\end{array}$ & $\begin{array}{c}133.3 \pm 91.6 \\
{[59,23-138,20-133]}\end{array}$ & $\begin{array}{c}54.0 \pm 15.0 \\
{[5,1-591,2-43]}\end{array}$ & $<0.001$ \\
\hline
\end{tabular}

These italic $P$ values are the sum $P$ values of the ages and of the type of VAD support. VAD, ventricular assist device.

weaning than those with postoperative HF, as shown in the univariate analysis. Multivariate analysis confirmed the significant advantage of lower age and found significant differences within the various diagnosis groups (Table 5).

\section{Discussion}

There has been no existing large series of myocardial recovery in children with HF supported with VAD. Neither 


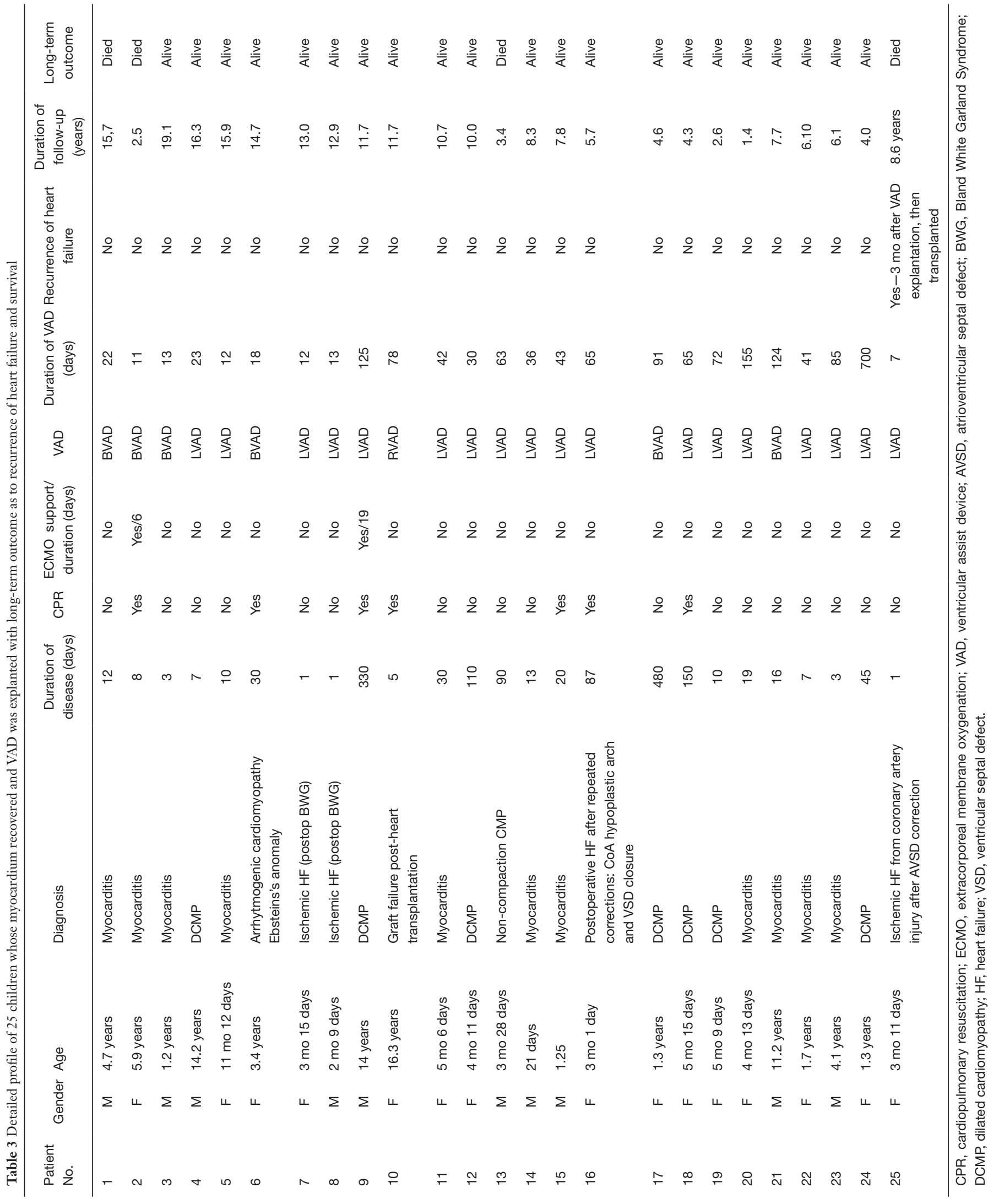




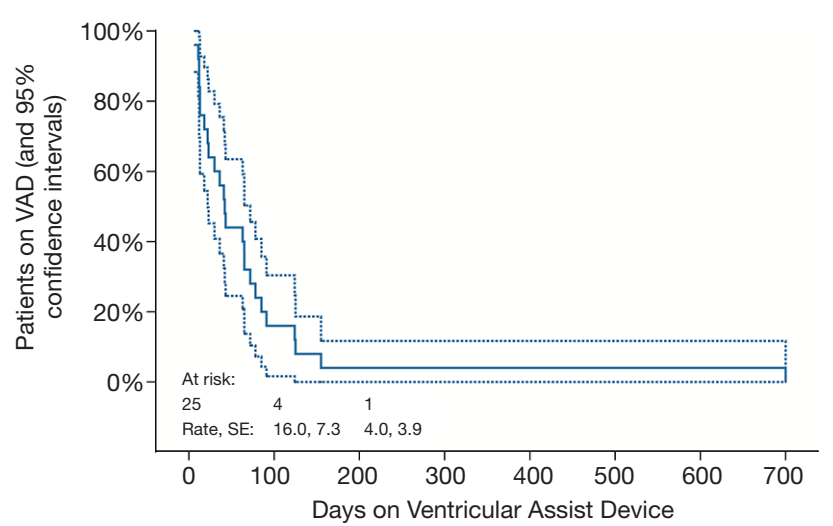

Figure 1 Time-related VAD support in patients who could be weaned. All patients who recovered were weaned within 155 days after VAD implementation, except one patient who recovered after 700 days. SE, standard error; VAD, ventricular assist device.

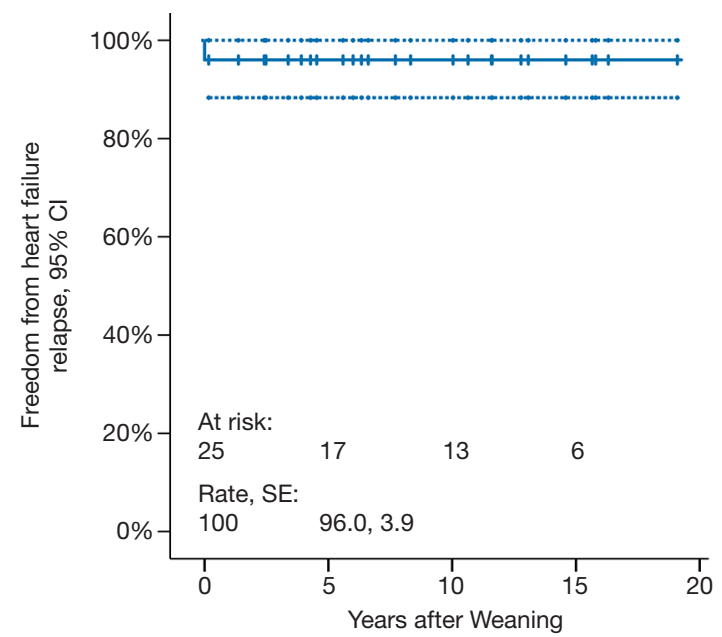

Figure 2 Freedom from recurrence of heart failure in children weaned off VAD support. All weaned patients remained free from heart failure recurrence, except one with non-compaction CMP who died after 61 days. SE, standard of error; CI, confidence interval.

have factors been definitely pinpointed which predict recovery nor is there certainty of recovery durability postweaning. Furthermore, reports on long-term outcomes of myocardial recovery after VAD support are sparse.

It is interesting to note that myocardial recovery occurred mostly in younger children. Myocardial recovery, seen in 12 $(48 \%)$ infants $(0-<1$ year) was sustainable except in one who had HF recurrence 3 months post-weaning. Younger age per se, a factor in myocardial plasticity $(2,4,11)$, may then

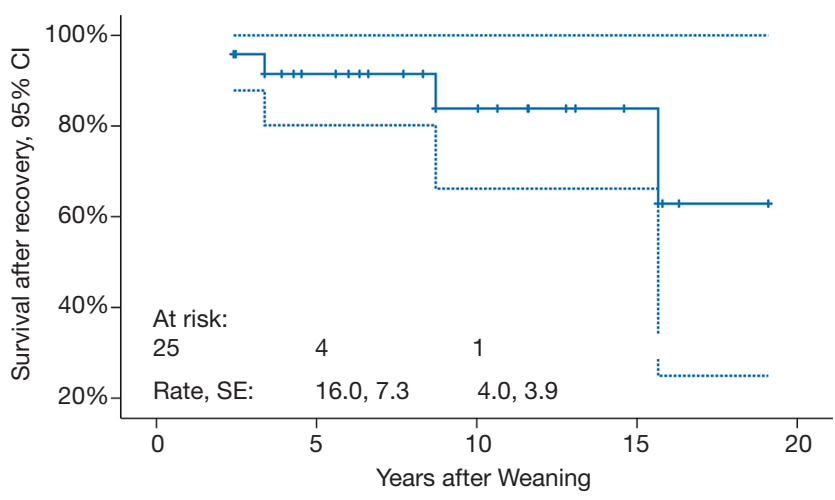

Figure 3 Cumulative survival of 25 children who had myocardial recovery showing a survival rate is $62.9 \% \pm 19.4 \%$ at 19 years. SE, standard of error; CI, confidence interval.

predict myocardial recovery. This strongly supports the report of Miera et al. (8) that positive predictors for recovery were younger age, with children $<2$ years having a 5.6fold higher chance of recovery and confirmed myocarditis. Reverse remodeling on VAD at a histological level is agedependent with an increased cardiomyocyte proliferation rate and decreased development of interstitial fibrosis in children $<2$ years of age (12).

The role of gender in HF has not been specified in children. Although substantial sex differences in LV mass, with males having consistently larger LV dimensions which relate to sex differences in genetic regulation have been reported (13), we found no impact of gender $(\mathrm{P}=0.183)$ on weaning outcome, long-term post-VAD survival and sustainability of myocardial recovery, even when stratified to age, diagnosis, and type of VAD support (9).

The nature and acuteness of myocardial injury greatly influence the type and degree of recovery. HF may present suddenly without any prior disease (acute HF) as in acute myocarditis, or maybe characterized by a prolonged time course with progressive deterioration in functional capacity (subacute and/or chronic HF), as in DCMP. Patients with acute HF have not been exposed to the deleterious effects of prolonged abnormal hemodynamics and neurohormonal perturbations; in contrast, longer duration of HF is associated with profound systemic changes which progress over time (14).

A salient finding in this study is the higher frequency of myocardial recovery after ventricular unloading in children with acute HF regardless of disease severity and use of 
Table 4 Comparative echocardiography-measured LV size, geometry and function pre- and during VAD support and after VAD explantation in LVAD and BiVAD supported children based on one-way ANOVA

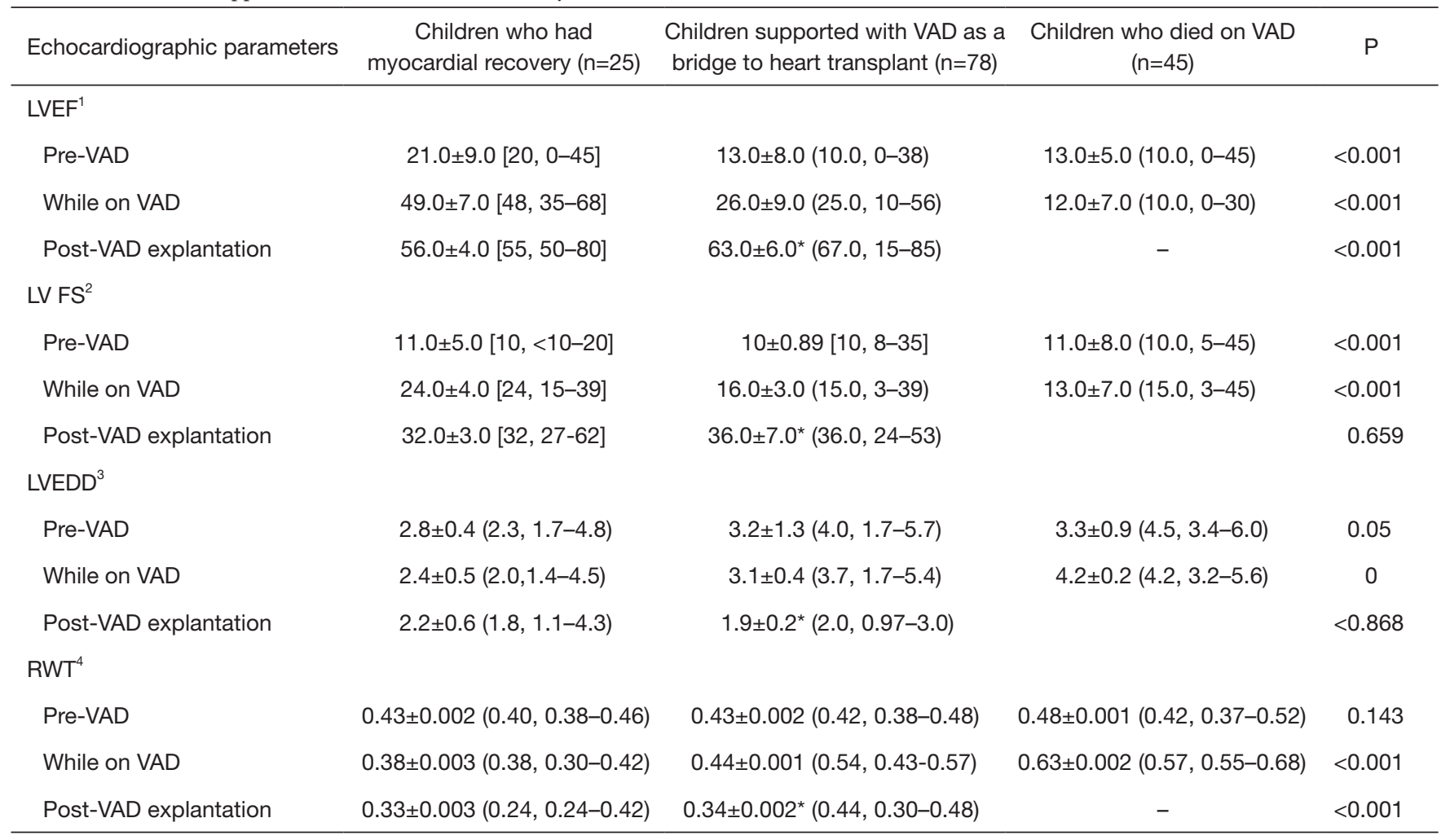

${ }^{1}$, left ventricular ejection fraction; ${ }^{2}$, left ventricular fractional shortening; ${ }^{3}$, left ventricular end-diastolic diameter; ${ }^{4}$, relative wall thickness $(2 \times$ posterior wall thickness/LVEDD); *, after transplantation; ANOVA-analysis of variance. LV, left ventricular; VAD, ventricular assist device.

biventricular support compared to those with HF symptoms $>1$ year. Children with shorter HF duration presumably may have more preserved cardiac reserve; hence, a greater likelihood to respond to VAD support.

All 33 children with myocarditis had biopsy-and immunohistochemistry-confirmed myocarditis ranging from acute or chronic to fulminant onset. Other possible causes of cardiomyopathy, i.e., bacterial and fungal infection, mitochondrial defects, storage disease, were considered and found negative in specific laboratory tests. Identified were adenovirus, cytomegalovirus, coxsackie virus, enterovirus, human herpes virus 6 and Parvovirus B19. Some of these children has been reported by our group in 1998 (2), 1999 (5), 2011 (6) and in 2018 (8). Although 2 patients died 2.4 and 15.7 years post-VAD explantation, none ever had HF recurrence. The survivors have no residual ventricular dysfunction 1.4-19.1 years post-weaning. This shows that children with myocardial dysfunction from myocarditis have reversible $\mathrm{HF}$ and can recover if the circulation is supported during the critical time (7-14 days) of inflammation and healing. Long-term follow-up has been necessary to rule out DCMP—a sequela in survivors of severe myocarditis $(15,16)$.

HF from CHD represents a different predicament $(17,18)$ than in those with infectious- or DCMP-related HF. Not only that they have complex anatomical and pathophysiological substrates and suffer from significant comorbidities, they also have undergone multiple surgeries and VAD implantation may be their fourth or fifth procedure. In 29 children with HF from CHD, only one (3-month old girl, HF ensued after repeated corrections of aortic coarctation and arch hypoplasia and ventricular septal defect closure, repeatedly resuscitated) supported with LVAD for 63 days recovered fully and is alive 5.7 years now.

In ischemic HF and hibernating myocardium, ventricular unloading improves coronary perfusion by decreasing cellular and tissue oedema, as shown in the 3 infants with postoperative ischemia. VAD support was short $(12,13$ and 7 days, respectively). The first two are alive 12.9 and 13 years post-VAD explantation. HF recurred in the third infant 3 months later. This raises a concern such that had 


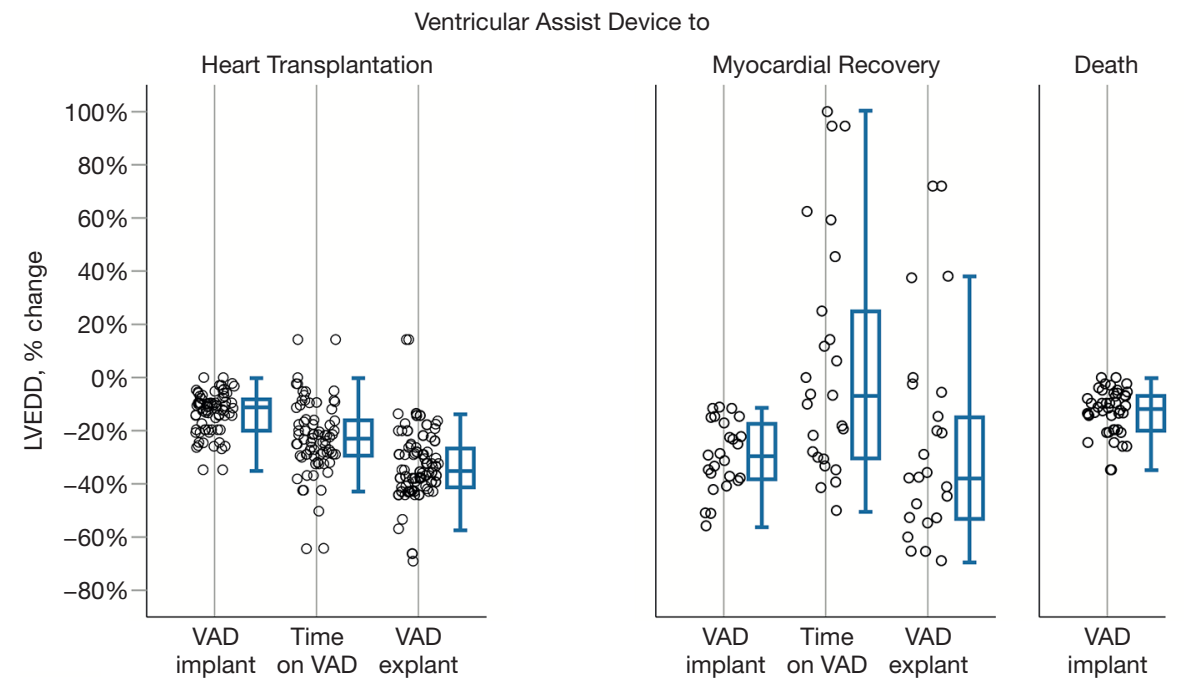

Figure 4 Percent change in left ventricular end diastolic diameters of three patient groups who had VAD support: those who underwent heart transplantation, recovered and died on VAD. The differences are shown in 3 time points: shortly before VAD implantation, shortly after VAD implantation and after VAD explantation for recovery-patients and after heart transplantation for heart transplant recipients. Patients who recovered had a more pronounced early LVEDD reduction after VAD implantation than those who needed transplantation or those who died. LVEDDs of transplant heart recipients remain smaller, while the LVEDD of patients who had myocardial recovery may either increase or decrease after VAD explantation. The plots show quartiles (boxes represent percentile 25 to 75 , the median is the horizontal line in the boxes, whiskers are maximal and minimal values, apart from outliers and extreme values) and individual differences. LVEDD, left ventricular end-diastolic diameter; VAD, ventricular assist device.

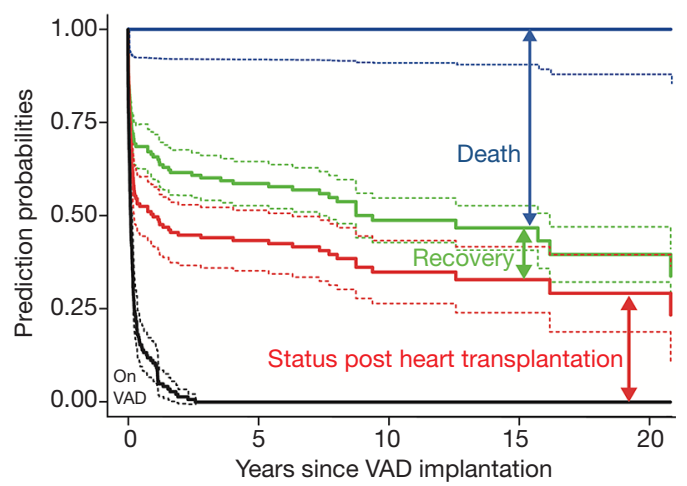

Figure 5 Stacked transition probabilities derived from multistate estimation for time-related events of children with heart failure supported with VAD. The continuous lines show the estimations, the dashed lines their confidence limits. Numbers of patients at risk in a certain state may be read from the previous figures; the model shown considers all survivors at the time shown in the $\mathrm{x}$-axis. The vertical distance between a line and the line underneath shows the fraction of patients that can be expected to be in the state described in the figure at the time given on the $\mathrm{x}$-axis. To find the percentage of patients that corresponds to the vertical distance, shift the distance arrow to the bottom of the $y$-axis on the bar top, the scale indicates the percentage of patients being in this state. For example, at 15 years about $13 \%$ of the children who needed VAD support were in the state of recovery. Risk factors for the transitions from one state to another are illustrated. State wise risk factors derived from this model are described in Table 5. VAD, ventricular assist device. 
Table 5 Univariate and multivariate analysis of risk factors in weaning from VAD

\begin{tabular}{|c|c|c|c|c|}
\hline Analysis & Factor/category & $\begin{array}{l}\text { Category comparisons/increment } \\
\text { effect: hazard ratio }\end{array}$ & \multicolumn{2}{|c|}{$P$ value } \\
\hline \multirow{4}{*}{ Univariate } & LVAD vs. BVAD & 1.2104 & 0.6881 & 0.6881 \\
\hline & Diagnosis & - & 0.0006 & - \\
\hline & Myocarditis vs. CHD & 4.2408 & - & 0.0699 \\
\hline & $\mathrm{POHF}$ vs. CHD & 7.4305 & - & 0.0247 \\
\hline \multirow[t]{4}{*}{ Multivariate } & Age (increment: years) & 0.8773 & 0.0074 & - \\
\hline & LVAD vs. BVAD & 0.8724 & 0.7910 & 0.7910 \\
\hline & Myocarditis vs. CHD & 4.7034 & - & 0.0541 \\
\hline & $\mathrm{POHF}$ vs. CHD & 4.8797 & - & 0.0865 \\
\hline
\end{tabular}

HR, hazard ratio; Age, age at VAD implantation (years); DCMP, dilated cardiomyopathy; CHD, congenital heart disease; POHF, postoperative heart failure.

the ventricular unloading been prolonged, myocardial recovery may have been sustained.

Acute graft failure may result from long ischaemic times, inadequate myocardial preservation during procurement, hyperacute rejection, or poor graft adaptation to the recipient's haemodynamic environment. ECMO, which supports the organ systems and allows the freshlytransplanted heart to work under less stressful conditions, maybe indicated when graft failure is due to a biventricular dysfunction. However, when there are clear signs of RV dysfunction, it is very important to support the failing RV with a VAD to avoid potentially irreversible graft injury (19).

Arrhythmogenic cardiomyopathy is a primary RV disorder in which fibrofatty infiltration of the RV wall leads to RV dilatation and systolic dysfunction (20). Insufficient blood supply leads to acute myocardial ischemia, with its inherent arrhythmogenic mechanisms (21). Ventricular unloading enhanced normalization of electrical conduction pathway, as shown in a 3-year-old girl with Ebstein anomaly who had supraventricular tachycardia and underwent CPR. With a markedly reduced RV function and persistence of arrhythmia despite anti-arrhythmic agents and repeated cardioversions, HF ensued. During the 18-day VAD support, her condition stabilized with disappearance of arrhythmia. She is still alive 14.7 years after VAD explantation.

Noteworthy is the observation that even in chronic HF, such as in DCMP, myocardial recovery occurred by unloading the ventricle with $\mathrm{VAD}$, as our group initially demonstrated in 1995 in five adults with idiopathic DCMP (20).

Ventricular dilation has been considered an irreversible aspect of ventricular remodeling in DCMP (22). Some reports suggested that restoration of fiber orientation and regression of myocyte hypertrophy, consistent with the marked reduction of ventricular mass may occur during long-term VAD support (23). Furthermore, if sufficient ventricular unloading is achieved, some aspects of cardiac remodeling can be reversed (24). This has been evidenced in the present study.

Device explantation in chronic cardiomyopathy has been rarely reported in children (25). Among 76 patients with DCMP in our series, we were able to wean 7 children after ventricular unloading ranging from 30-700 days. These children with stable recovery (2.6-16.3 years post-VAD explantation) were younger and had shorter (7-480 months) HF duration before VAD implantation. Less chronicallyaltered hearts appeared to have a better prospect to recover. With this small number of patients, it still remains difficult to prospectively identify those with DCMP who would be weaned off VAD support and will have optimal and stable 
cardiac recovery.

Since 1992 (26), we use rescue ECMO support in children with $\mathrm{HF}$ especially in those with potential for fast myocardial recovery and expected short-term support. ECMO is switched to VAD when longer-term support is needed and the patient had not suffered severe organ damage before or during ECMO support. Another indication to switch to VAD is continuous bleeding on ECMO, because of the lower anticoagulation required with VAD (2). Among 49 children who suffered cardiac arrest, ECMO was initially implanted in 16 before switching to VAD. It is shown in this study that cardiac arrest significantly influences the outcome of VAD support $(\mathrm{P}<0.001)$. In contrast, neither the use of ECMO $(\mathrm{P}=0.56)$ nor its duration of support $(\mathrm{P}=0.568)$ prior to VAD implantation predict recovery or death on VAD.

Although it may often be concluded that VAD implantation was not absolutely necessary and ECMO would have been very likely sufficiently, pre-implant prediction of cardiac improvement during mechanical circulatory support is not reliable, neither in young patients nor in those with short duration of HF, although in those patients the incidence of recovery is theoretically higher. As we have shown in Tables 3,4 (2 with BVAD and 2 with LVAD) of the 25 successfully explanted patients had indeed a VAD support of only 12-13 days. In those patients it may be assumed that ECMO would have been sufficient, but at the time of VAD implantation, short-term recovery was not reliably predictable and there have been some arguments favouring VAD implantation.

It is not contested that the use of ECMO, especially postoperatively, has been shown to be lifesaving in children with myocardial failure. However, when pulmonary function is normal, ECMO is not always the optimal method for prolonged circulatory support because of complexity of equipment, bleeding complications, capillary leak syndrome, and non-pulsatile flow. The advantages of ECMO in contrast to BVAD are easier cannulation, oxygenator support in pulmonary insufficiency, and the possibility of slow reduction of circulatory support. While bleeding related to heparinization is the most common complication of ECMO related to heparinization, BVAD limits haemorrhagic complications as less anticoagulation with heparin is required. The advantages of BVAD are a much longer time gain to restore organ function, elimination of oedema, extubation, mobilisation, improvement in nutrition, and allowing the children to regain consciousness to assess neurological status. Reduced capillary leak in BVAD may be due to the pulsatile flow and to less contact with synthetic surfaces.

It is acknowledged that patients necessitating BVAD support have poorer outcomes than those requiring isolated LVAD support. This present study has shown that the number of ventricles supported does not predict the outcome (recovery vs. death) of ventricular unloading $(\mathrm{P}=0.566)$.

Animal models of unloading the non-failing myocardium by LVAD (27) suggested that prolonged unloading leads to cardiomyocyte atrophy making the heart not strong enough to be reloaded, which might affect the durability and sustainability of recovery. Moreover, an animal study of failing hypertrophied hearts indicated that unloading resulted in a decrease of cardiac myocytes in size beyond normal values (28). In human studies, electron microscopy suggested no evidence of cardiomyocyte atrophy or degeneration even with $>6$ months of LVAD support (29). Studies with shorter periods of unloading were not associated with contractile dysfunction $(29,30)$. These suggest that adverse remodeling could sometimes occur with very prolonged unloading.

The chances of successful weaning with long-term freedom from $\mathrm{HF}$ recurrence are low, even if regression of LV dilation and improvement of LVEF reach acceptable levels for a possible LVAD explantation after $\geq 6$ months of VAD support. This has been seen in patients with LVAD implanted as bridge to heart transplantation, in those with prolonged support waiting for cardiac recovery, in patients with persistence of massive LV dilation and low off-pump LVEF associated with a long pre-implant HF history and especially in patients with chronic cardiomyopathy. In adults, it has been demonstrated that in LVAD recipients with off-pump LVEF $<45 \%$ and a pre-implant duration of cardiac disease of $>5$ years have nearly no chances to remain more than 3 years free from post-explant HF recurrence. In that patients with persistent $\mathrm{LV}$ dilation, the predictive value for post-explant $\mathrm{HF}$ recurrence before the end of the 3 rd post-explant year can reach $90 \%$ if the pre-explant offpump LVEF is $\leq 50 \%$ (30). These data suggest that for each patient a careful evaluation of both risks and chances of a prolongation of the VAD support is preferable.

The children who had myocardial recovery have had VAD support ranging from 7-700 days, longer than those children bridged to transplant (range, 23-133 days). This uptrends a great concern that myocardial recovery may have been achieved had this latter group been supported longer with VAD. However, with our weaning strategy, we 
believe we have not missed indices of recovery, and that transplantation in those who did not recover, was inevitable. This study has shown that indeed, duration of VAD support may predict a potential to myocardial recovery $(\mathrm{P}<0.001)$.

All children in this series presented with a markedly reduced EF. Those who were weaned off exhibited remarkable improvement in LVEF $(\mathrm{P}<0.01)$ than those who were eventually transplanted. This is because the latter group with insufficient remodeling and less functional improvement had DCMP with a longer HF duration. Using a univariate analysis, LVEF proved to be a highly objective parameter to predict myocardial recovery.

Recovery rates are higher in children with less ventricular dilation, and a less altered $L V$ contractile function may be easily reversible, as reported for adult patients $(23,28,29)$, although these changes are not always associated with clinically-relevant recovery $(28,29)$ VAD can induce reverse remodeling through reversal of myocyte and extracellular matrix abnormalities, followed by reversal of ventricular dilation and dysfunction. These beneficial effects incorporate a series of integrated changes in myocyte structure and function, as well as modifications in ventricular structure and organization accompanied by shifts of the ventricular end-diastolic pressure-volume relationship toward normal. Presently, however, neither the components of the reverse remodeling process which are necessary for myocardial recovery, nor the minimum levels of reverse remodeling necessary to allow VAD removal are sufficiently well known (31). We do not use myocardial histology as a criterion for weaning decisions. Only patients with acute myocarditis or severe cardiac allograft rejection as the underlying cause for drug-refractory HF underwent myocardial biopsy (to confirm the absence of myocarditis or rejection) before VAD removal.

Because FS measures myocardial function just in one plane and describes the contractile function in only a certain region of two ventricular walls, it has a more limited clinical use than the LVEF. Despite its less important role for weaning decisions, it is a useful parameter for selection of potential weaning candidates.

Remodeling patterns of the $\mathrm{LV}$ can be assessed by $\mathrm{LV}$ geometry measurement through RWT and maybe broadly categorized as normal or adverse remodeling, either eccentric (low RWT) or concentric (high RWT) (32). Data on the relationship between the magnitude of eccentric or concentric hypertrophy in children is nil. In this series, children with low RWT were younger and had more advanced HF, hence, lower LVEF than those with high RWT. Myocyte hypertrophy with wall fibrosis is pronounced in children with DCMP; hence, despite ventricular unloading, RWT may be similar to the pre-VAD RWT. In myocarditis; RWT correlates indirectly with wall thickness and inversely with LVEDD. Thus, the magnitude of RWT cannot mirror the extent of myocyte hypertrophy nor myocyte edema.

In the current study, both wall thickness (measure of wall fibrosis) and diastolic diameter (measure of remodeling) draw attention to their potential to predict myocardial recovery $(\mathrm{P}<0.001)$ even in children with marked $\mathrm{LV}$ dilation at presentation. This contributes new information.

The question remains as to whether these children are still at risk for recurrent HF. Birks (33) proposed strategies to thicken the myocardium to enhance the durability of recovery before explantation, such as clenbuterol administration or intermittently reducing the pump speed and pulsatilely load the heart.

Be it as it may, further understanding of myocardial recovery in response to VAD support in children must be intensively addressed in future research.

\section{Limitations of the study}

This is a retrospective study based on institutional databases with the inherent limitations of data entries. Stratification by duration of HF was based on retrospective review of medical records. It is possible that those patients classified as AHF could either represent true new-onset HF or chronic HF with an acute decompensation not previously recognized.

\section{Conclusions}

This study focused on the weaning outcomes of children with HF from various etiologies supported with VAD to effect myocardial recovery. Post-weaning myocardial recovery and cardiac stability appeared durable over longterm. Young patients with short duration of HF and less myocardial fibrosis are more likely to recover. Absence of cardiac arrest, prolonged VAD support and cardiac size, geometry and function may identify patients who may likely have myocardial recovery.

\section{Acknowledgments}

We sincerely appreciate the assistance of Prof. Dr. rer. nat. Hartmut Hecker from the Institute of Biometrie, Hannover 
Medical School, in developing the competing risk analysis model. We thank the student research assistants from the Universitätsmedizin Berlin Charite for follow-up data collection.

Funding: None.

\section{Footnote}

Provenance and Peer Review: This article was commissioned by the editorial office, Cardiovascular Diagnosis and Therapy for the series "Heart Failure in the Young and Old: Insights into Various Therapies". The article has undergone external peer review.

Data Sharing Statement: Available at http://dx.doi. org/10.21037/cdt-20-278

Conflicts of Interest: All authors have completed the ICMJE uniform disclosure forms (available at http://dx.doi. org/10.21037/cdt-20-278). The series "Heart Failure in the Young and Old: Insights into Various Therapies" was commissioned by the editorial office without any funding or sponsorship. RH served as the unpaid Guest Editor of the series and serves as an unpaid editorial board member of Cardiovascular Diagnosis and Therapy from July 2019 to Jun 2021. The authors have no other conflicts of interest to declare.

Ethical Statement: All authors have worked on this paper and are accountable for all aspects of the work in ensuring that questions related to the accuracy or integrity of any part of the work are appropriately investigated and resolved. The Institutional Review Board approved this study and waived the need for patients' consent as the study itself was regarded as non-interventional and data protection standards were fulfilled.

Open Access Statement: This is an Open Access article distributed in accordance with the Creative Commons Attribution-NonCommercial-NoDerivs 4.0 International License (CC BY-NC-ND 4.0), which permits the noncommercial replication and distribution of the article with the strict proviso that no changes or edits are made and the original work is properly cited (including links to both the formal publication through the relevant DOI and the license). See: https://creativecommons.org/licenses/by-nc-nd/4.0/.

\section{References}

1. Hetzer R, Kaufmann F, Delmo Walter EM. Paediatric mechanical circulatory support with Berlin Heart EXCOR: development and outcome of a 23-year experience. Eur J Cardiothorac Surg 2016;50:203-10.

2. Hetzer R, Potapov E, Alexi-Meskishvili V, Weng Y, et al. Single-center experience with treatment of cardiogenic shock in children by pediatric ventricular assist devices. J Thorac Cardiovasc Surg 2011;141:616-23.

3. Dandel M, Knosalla C, Hetzer R. Contribution of ventricular assist devices to the recovery of failing hearts: a review and the Berlin Heart Center Experience. Eur J Heart Fail 2014;16:248-63.

4. Dandel M, Hetzer R. Myocardial recovery during mechanical circulatory support: long-term outcome and elective ventricular assist device implantation to promote recovery as a treatment goal. Heart Lung Vessel 2015;7:289-96.

5. Hetzer R, Müller J, Weng Y, et al. Cardiac recovery in dilated cardiomyopathy by unloading with a left ventricular assist device. Ann Thorac Surg 1999;68:742-9.

6. Hetzer R, Loebe M, Potapov EV, et al. Circulatory support with pneumatic paracorporeal ventricular assist device in infants and children. Ann Thorac Surg 1998;66:1498-506.

7. Stiller B, Dähnert I, Weng YG, et al. Children may survive severe myocarditis with prolonged use of biventricular assist devices. Heart 1999;82:237-40.

8. Miera O, Germann M, Cho MY, et al. Bridge to recovery in children on ventricular assist devices-protocol, predictors of recovery, and long-term follow-up. J Heart Lung Transplant 2018;37:1459-66.

9. Hetzer R, Javier MFDM, Delmo Walter EM. Role of paediatric assist device in bridge to transplant. Ann Cardiothorac Surg 2018;7:82-98.

10. Hetzer R, Alexi-Meskishvili V, Weng Y, et al. Mechanical cardiac support in the young with the Berlin Heart EXCOR pulsatile ventricular assist device: 15 years' experience. Semin Thorac Cardiovasc Surg Pediatr Card Surg Annu 2006;9:99-108.

11. Birks EJ. Myocardial recovery in patients with chronic heart failure: is it real? J Card Surg 2010;25:472-7.

12. Kasten J, Rakheja D, Zhang S, et al. Reverse histologic remodeling after mechanical unloading of failing hearts in children with dilated cardiomyopathy. J Heart Lung Transplant 2017;36:1268-71. 
13. Goble MM, Mosteller M, Moskowitz WB, et al. Sex differences in the determinants of left ventricular mass in childhood. The Medical College of Virginia Twin Study. Circulation 1992;85:1661-5.

14. Loyaga-Rendon RY, Acharya D, Pamboukian SV, et al. Duration of Heart Failure Is an Important Predictor of Outcomes After Mechanical Circulatory Support. Circ Heart Fail 2015;8:953-9.

15. Kereiakes DJ, Parmley WW. Myocarditis and cardiomyopathy. Am Heart J 1984;108:1318-26.

16. Gagliardi MG, Bevilacqua M, Squitieri C, et al. Dilated cardiomyopathy caused by acute myocarditis in pediatric patients: evolution of myocardial damage in a group of potential heart transplant candidates. J Heart Lung Transplant 1993;12:S224-9.

17. Miller JR, Eghtesady P. Ventricular assist device use in congenital heart disease with a comparison to heart transplant. J Comp Eff Res 2014;3:533-46.

18. Fan Y, Weng YG, Huebler M, et al. Predictors of inhospital mortality in children after long-term ventricular assist device insertion. J Am Coll Cardiol 2011;58:1183-90.

19. Hetzer R, Delmo Walter EM. Rescue mechanical circulatory support for failing transplanted hearts. Eur J Cardiothorac Surg 2012;42:702-3.

20. Müller J, Wallukat G, Weng YG, et al. Weaning from mechanical cardiac support in patients with idiopathic dilated cardiomyopathy. Circulation 1997;96:542-9.

21. Coronel R, Wilders R, Verkerk AO, et al. Electrophysiological changes in heart failure and their implications for arrhythmogenesis. Biochim Biophys Acta 2013;1832:2432-41.

22. Levin HR, Oz MC, Chen JM, et al. Reversal of chronic ventricular dilation in patients with end-stage cardiomyopathy by prolonged mechanical unloading. Circulation. 1995;91:2717-20.

23. Jacquet L, Zerbe T, Stein K, et al. Evolution of human cardiac myocyte dimension during prolonged mechanical support. J Thorac Cardiovasc Surg 1991;101:256-9.

24. Dandel M, Weng Y, Siniawski H, et al. Long-term results in patients with idiopathic dilated cardiomyopathy after weaning from left ventricular assist devices. Circulation 2005;112:I37-45.

25. Jefferies JL, Morales DL. Mechanical circulatory support in children: bridge to transplant versus recovery. Curr Heart Fail Rep 2012;9:236-43.

26. Delmo Walter EM, Alexi-Meskishvili V, Huebler M, et al. Rescue extracorporeal membrane oxygenation in children with refractory cardiac arrest. Interact Cardiovasc Thorac Surg 2011;12:929-34.

27. Kinoshita M, Takano H, Takaichi S, et al. Influence of prolonged ventricular assistance on myocardial histopathology in intact heart. Ann Thorac Surg 1996;61:640-5.

28. Soppa GK, Lee J, Stagg MA, et al. Prolonged mechanical unloading reduces myofilament sensitivity to calcium and sarcoplasmic reticulum calcium uptake leading to contractile dysfunction. J Heart Lung Transplant 2008;27:882-9.

29. Birks EJ. Molecular changes after left ventricular assist device support for heart failure. Circ Res 2013;113:777-91

30. Dandel M, Weng Y, Siniawski H, et al. Heart failure reversal by ventricular unloading in patients with chronic cardiomyopathy: Criteria for weaning from ventricular assist devices. Eur Heart J 2011;32:1148-60.

31. Mann DL, Barger MP, Burkhoff D. Myocardial recovery and the failing heart. J Am Coll Cardiol 2012;60:2465-72.

32. Biton Y, Goldenberg I, Kutyifa V, et al. Relative Wall Thickness and the Risk for Ventricular Tachyarrhythmias in Patients With Left Ventricular Dysfunction. J Am Coll Cardiol 2016;67:303-12.

33. Birks EJ. The Promise of Recovery. JACC Heart Fail 2016;4:577-9.
Cite this article as: Javier Delmo EM, Javier MFDM, Böthig D, Rüffer A, Cesnjevar R, Dandel M, Hetzer R. Heart failure in the young: Insights into myocardial recovery with ventricular assist device support. Cardiovasc Diagn Ther 2021;11(1):148163. doi: $10.21037 / \mathrm{cdt}-20-278$ 